\title{
Reminders reinstate context-specificity to generalized remote memories in rats: relation to activity in the hippocampus and aCC
}

\author{
Melanie J. Sekeres, ${ }^{1}$ Morris Moscovitch, ${ }^{2,3,4}$ Cheryl L. Grady, ${ }^{2,4,5}$ D. Gregory Sullens, ${ }^{1}$ \\ and Gordon Winocur ${ }^{2,4,5,6}$ \\ ${ }^{1}$ Department of Psychology and Neuroscience, Baylor University, Waco, Texas 76798, USA; ${ }^{2}$ Rotman Research Institute, Baycrest, \\ Toronto, Ontario M6A 2E1, Canada; ${ }^{3}$ Department of Psychology, Baycrest, Toronto, Ontario M6A 2E1, Canada; ${ }^{4}$ Department of \\ Psychology; ${ }^{5}$ Department of Psychiatry, University of Toronto, Toronto, Ontario M5S 3G3, Canada; ${ }^{6}$ Department of Psychology, \\ Trent University, Peterborough, Ontario K9J 7B8, Canada
}

\begin{abstract}
Conditioned fear memories that are context-specific shortly after conditioning generalize over time. We exposed rats to a context reminder $30 \mathrm{~d}$ after conditioning, which served to reinstate context-specificity, and investigated how this reminder alters retrieval-induced activity in the hippocampus and anterior cingulate cortex $(\mathrm{aCC})$ relative to a no reminder condition. c-Fos expression in dorsal CAl was observed following retrieval in the original context, but not in a novel context, whether or not the memory was reactivated, suggesting that dCAl retains the context-specific representation. c-Fos was highly expressed in aCC following remote memory testing in both contexts, regardless of reminder condition, indicating that aCC develops generalized representations that are insensitive to memory reactivation.
\end{abstract}

[Supplemental material is available for this article.]

Over time, memories in rats that were once highly specific to the contextual cues present during memory acquisition are changed as the memory transforms from one that is critically dependent upon the hippocampus, to one that becomes supported by both the hippocampus and the prefrontal cortex, particularly anterior cingulate cortex (aCC) (Frankland et al. 2004; Wiltgen and Silva 2007; Winocur et al. 2007; Einarsson and Nader, 2012; Wheeler et al. 2013; Cullen et al. 2015; Einarsson et al. 2015; Kitamura et al. 2017; Vetere et al. 2017; Sekeres et al. 2018a; DeNardo et al. 2019; Ortiz et al. 2019). Reactivating an established $30 \mathrm{~d}$ old fear memory by presenting the contextual cues that were present during encoding reinstates context-specificity to the remote memory (Sekeres et al. 2012), and renders the memory sensitive to hippocampal disruption during the postreactivation reconsolidation window (Debiec et al. 2002). Lesioning the hippocampus after a context reminder of a remote memory abolishes both context specific and generalized context memories (Winocur et al. 2009). These findings support the notion that different types of memory can dynamically coexist in the brain. Which type is ultimately expressed during retrieval likely depends on the availability of the memory trace, and upon the adaptiveness of being able to access a specific memory given the situational demands at the time of retrieval.

Temporary pharmacological inactivation of both the dorsal hippocampus and the aCC following a context reminder is required to impair memory retrieval in the original context (Einarsson et al. 2015). Rapid optogenetic inactivation of the hippocampal dorsal CA1 (dCA1) neurons, however, is sufficient to impair retrieval of a remote context memory (Goshen et al. 2011), indicating that the default remote context representation is the hippocampal-dependent memory. These loss-of-function studies suggest that reactivating the remote context memory reengages the hippocampal-dependent context memory, and suppresses retrieval of the generalized, nonhippocampal (presumably cortical) memory trace (Winocur et al. 2009). If unavailable, the less precise cortical representation of the memory may slowly come online to support retrieval. In this case, disruption of both the dorsal hippocampus and the aCC is required to impair memory retrieval (Einarsson et al. 2015).

It remains unknown how aCC-hippocampal patterns of activity interact at the cellular level in the intact brain to support retrieval of a recently reactivated remote context memory under natural conditions (for reviews, see Hardt and Nadel 2018; Sekeres et al. $2018 b)$. Considerable evidence suggests that reactivating a remote fear memory should result in high hippocampal activity following retrieval in the original conditioning context but not in a novel context (Wiltgen et al. 2010; Sekeres et al. 2018a). With respect to the aCC, two outcomes are possible. The first is based on evidence that aCC activity, and the type of memory representation it develops over time, are insensitive to differences in retrieval context (Cullen et al. 2015; Einarsson et al. 2015; Sekeres et al. 2018a). It follows from this that the aCC should exhibit relatively low activity levels following retrieval of the recently reactivated memory. The second, based on our group's previous results, is that aCC activity is high during remote memory retrieval in either context, as the reorganized memory trace in the cortex comes online to support the generalized remote memory representation in the healthy brain under normal physiological conditions (Sekeres et al. 2018a).
Corresponding author: melanie_sekeres@baylor.edu

Article is online at http://www.learnmem.org/cgi/doi/10.1101/lm.050161. 119. Freely available online through the Learning \& Memory Open Access option.
(C) 2020 Sekeres et al. This article, published in Learning \& Memory, is available under a Creative Commons License (Attribution-NonCommercial 4.0 International), as described at http://creativecommons.org/licenses/by-nc/4.0/. 
To resolve this issue, we imaged immediate-early gene (IEG) expression of c-Fos in the dCA1 hippocampal subregion and the aCC following retrieval of a recently reactivated remote context fear memory in rats. Three month old rats were randomly assigned to four experimental conditions: No Reminder, Context-A test (NR-A, $n=10)$; No Reminder, Context-B test (NR-B, $n=10$ ); Reminder, Context-A test (R-A, $n=10)$; Reminder, Context-B test (R-B, $n=10)$. See Figure 1A for a schematic of the study design. Following previously reported procedures (Winocur et al. 2007, 2009, 2013; Sekeres et al. 2018a), each rat was given a 30 min context preexposure session in the conditioning chamber. Twentyfour hours later, each rat was given ten tone-shock pairings (tone: $2000 \mathrm{~Hz}$; $90 \mathrm{~dB}, 30 \mathrm{sec}$; shock: $1.5 \mathrm{~mA}, 1 \mathrm{sec}$ ) in the conditioning chamber (Context-A). Thirty days later, rats in the reminder (R) condition were individually placed back in the conditioning chamber for $60 \mathrm{sec}$ to reactivate the context memory. Five hours later, rats were individually tested either back in Context-A or in novel Context-B for $8 \mathrm{~min}$. A separate group of nonreminded (NR) rats underwent identical conditioning and testing procedures, but were not exposed to the $60 \mathrm{sec}$ reminder prior to test. All procedures were approved by Trent University's Animal Care Committee, and conducted in accordance with the guidelines set by the Canadian Council on Animal Care. Total time spent freezing during the reminder and test sessions was recorded. A home cage control group $(n=8)$ was included to control for baseline expression of c-Fos, a transcriptional regulator and marker of neuronal activity (Cole and Josselyn 2008; Cruz et al. 2015). These rats were maintained in their home cages throughout the experiment, and did not undergo any behavioral training or testing.

Immediately following testing, rats were transferred to a quiet, dark holding room. Ninety minutes following test (or home cage) conditions, rats were anesthetized and intracardially perfused with
PBS and 4\% paraformaldehyde (PFA). Brains were postfixed in PFA for $24 \mathrm{~h}$ at $4^{\circ} \mathrm{C}$. Brains were sectioned coronally (30 $\mu \mathrm{m}$ slices). For the aCC, 4-12 sections ranging between 1.70 and $-0.92 \mathrm{~mm} \mathrm{~A} / \mathrm{P}$ relative to bregma (Paxinos and Watson 1997) were randomly sampled and counted per brain (mean of 8.42, SD 2.2 sections per brain) for immunohistochemical analysis of c-Fos protein. For the hippocampus, $4-10$ sections ranging between -2.30 and $-5.60 \mathrm{~mm} \mathrm{~A} / \mathrm{P}$ were sampled and counted per brain (mean of 6.28 , SD 2.05 sections per brain). Sections were washed in PBS, then incubated with rabbit anti-c-Fos polyclonal primary antibody (1:1000, PBS and $0.3 \%$ Triton X-100, Calbiochem) at $4^{\circ} \mathrm{C}$ for $48 \mathrm{~h}$. Sections were then incubated with donkey-anti-rabbit Alexa 568 secondary antibody (1:200, Molecular Probes) for $2 \mathrm{~h}$ at room temperature, then washed and mounted with PermaFluor mounting medium (Thermo Scientific) on glass slides, and coverslipped.

Stained sections were analyzed using a fluorescent microscope (Nikon, MBA 92010 Eclipse NI). Digital images were taken at 10x magnification and stitched together using NIS-Elements software (Nikon, version 4.1.3) software to reconstruct each region of interest (dCA1, aCC). c-Fos positive nuclei in each subregion were manually counted using ImageJ (RRID: SCR_003070). The total number of c-Fos positive cells per region of interest was divided by the outlined area to generate a normalized cells/ area value. c-Fos expression was analyzed for home cage control brains to determine baseline c-Fos expression levels in each region of interest. Values of c-Fos positive cells from each experimental condition (NR-A, NR-B, R-A, R-B) were expressed as a percentage change relative to home cage control values. ANOVAs were conducted for c-Fos expression levels and for freezing behavior. Experimenters were blind to experimental conditions for all behavioral testing and histological analyses. All behavioral and

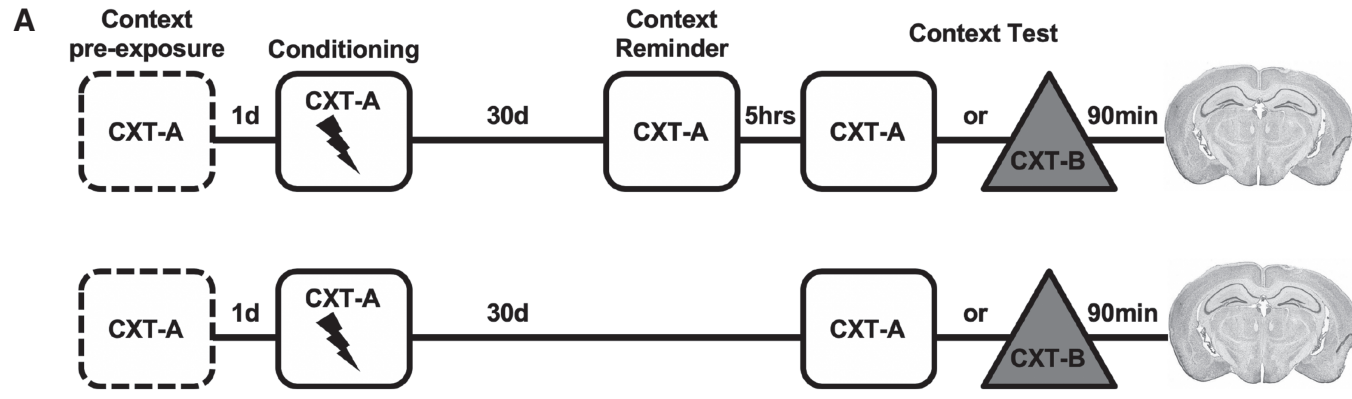

B

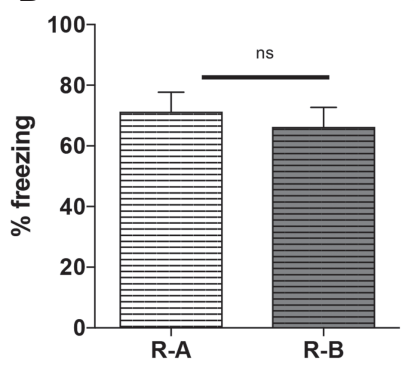

C

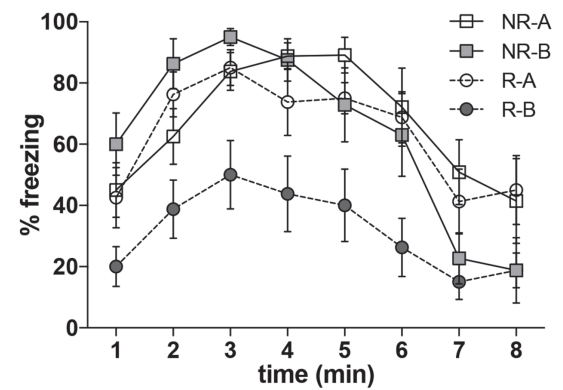

D

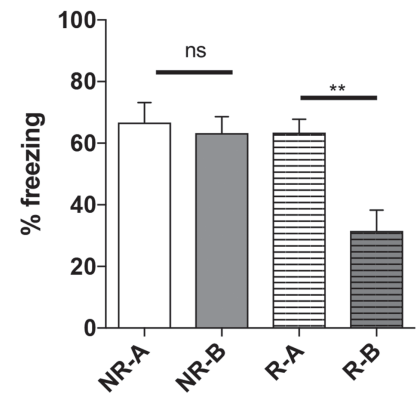

Figure 1. Reminder reinstates context-specificity to remote memory. (A) Experimental timeline for the Reminder (top) and for the NonReminder conditions (bottom). (B) Mean percent time freezing during the $60 \mathrm{sec}$ reminder. Rats subsequently tested in Context-A (R-A) and those subsequently tested in Context-B (R-B) displayed comparable levels of freezing during the reminder. (C) Freezing time course per group across the 8 min context fear memory test. $(D)$ Mean percent freezing per group during the $8 \mathrm{~min}$ context fear memory test. Rats froze significantly more in Context-A than in Context-B following a context reminder, but froze at similarly high levels in both Context-A and Context-B when no reminder was given prior to test. Error bars represent the SEM. ${ }^{(* *)} P<0.001$, ns $P>0.05$. 
histological statistical analyses were conducted using SPSS 25 (RRID: SCR_002865). See Supplemental Materials for further methodological detail.

Critically, no difference in freezing behavior was observed during the $60 \mathrm{sec}$ reminder session. Rats later tested in Context-A and those later tested in Context-B displayed equivalent high rates of freezing behavior during the reminder $\left(t_{(18)}=0.547, P=0.591\right.$; Fig. 1B). This result confirms that all rats retained memory for the conditioning context after $30 \mathrm{~d}$. Five hours later, freezing was assessed over an $8 \mathrm{~min}$ test period in either test context (Fig. 1C). A $2 \times 2$ ANOVA assessed mean freezing behavior during the test, with Context (Context-A, Context-B) and Reminder (R, NR) as between-subject factors. Significant main effects were found for Context, with more freezing in Context-A than in Context-B $\left(F_{(1,36)}=11.945, P=0.001, \eta_{\mathrm{p}}^{2}=0.249\right)$, and for Reminder, with more overall freezing during nonreminded trials $\left(F_{(1,36)}=11.704\right.$, $\left.P=0.002, \eta_{\mathrm{p}}^{2}=0.245\right)$. A significant Context $\times$ Reminder interaction $\left(F_{(1,36)}=7.746, P=0.009, \eta_{\mathrm{p}}^{2}=0.177\right)$ confirmed that rats exhibited context-specific memory following a reminder, freezing at higher levels in Context-A than Context-B (Fig. 1D, right). For nonreminded trials, groups displayed equivalent levels of freezing in both contexts (Fig. 1D, left), indicative of memory generalization in the novel context. In a previous study, we demonstrated that brief exposure to Context-B as a "reminder" prior to remote memory testing still led to high freezing in both Context-A and in Context-B (Winocur et al. 2009). Thus, the low freezing in Context-B observed following a Context-A reminder is likely reinstating context-specificity to the remote memory that is not observed in the absence of reactivation.

We next analyzed the expression of c-Fos in the dCA1 and the aCC (Fig. 2C). We waited $5 \mathrm{~h}$ between reminder and test to be with- in the $6 \mathrm{~h}$ window for cellular reconsolidation of remote context memory (Debiec et al. 2002; Sekeres et al. 2012), but outside of the activity-induced c-Fos expression time frame following the reminder session. IEG expression of c-Fos protein peaks 60-90 min following activation and returns to basal levels by $180 \mathrm{~min}$ postactivation (Morgan et al. 1987; Guzowski et al. 2001). Therefore, c-Fos expression levels reflect activity supporting memory retrieval during the test session, but not during the reminder session (see Supplemental Results).

For dCA1, a $2 \times 2$ ANOVA revealed a significant main effect of Context, with higher c-Fos levels following testing in Context-A than in Context-B $\left(F_{(1,28)}=8.535, P=0.007, \eta_{\mathrm{p}}^{2}=0.234\right)$, but no significant main effect of Reminder $\left(F_{(1,28)}=0.599, P=0.445, \eta_{\mathrm{p}}^{2}=\right.$ $0.021)$, and no Context $\times$ Reminder interaction $\left(F_{(1,28)}=0.973, P=\right.$ $0.332, \eta_{\mathrm{p}}^{2}=0.034$; Fig. $2 \mathrm{~A}$ ). A similar $2 \times 2$ ANOVA to assess c-Fos expression in the aCC revealed no significant main effects of Context $\left(F_{(1,29)}=1.050, P=0.314, \eta_{\mathrm{p}}^{2}=0.035\right)$ or $\operatorname{Reminder}\left(F_{(1,29)}=0.980, P=\right.$ $\left.0.330, \eta_{\mathrm{p}}^{2}=0.033\right)$, and no Context $\times$ Reminder interaction $\left(F_{(1,29)}=\right.$ 1.645, $P=0.210, \eta_{\mathrm{p}}^{2}=0.054$; Fig. $2 \mathrm{~B}$ ).

We previously assessed the time-dependent shift in retrievalassociated c-Fos expression within the dorsal hippocampus and aCC nodes of the context fear memory network, finding that the hippocampus was sensitive to context-specificity at both short and long delays, whereas the aCC was insensitive to the differences in testing context at either time point (Sekeres et al. 2018a). Based on these results, and our previous findings that a Context-A reminder, but not a Context-B reminder, after $30 \mathrm{~d}$ reinstates hippocampal-dependency to the remote memory (Winocur et al. 2009), we predicted that, in the intact brain, the hippocampus would continue to be recruited for context-specific remote memory retrieval.
A
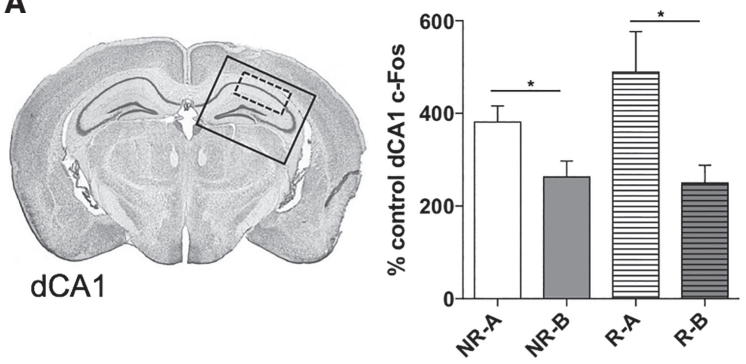

C
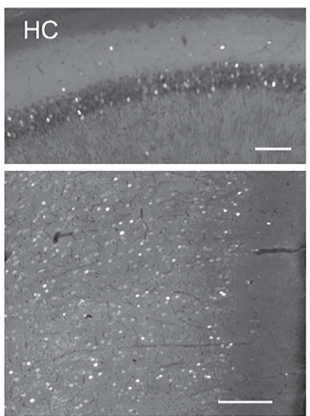
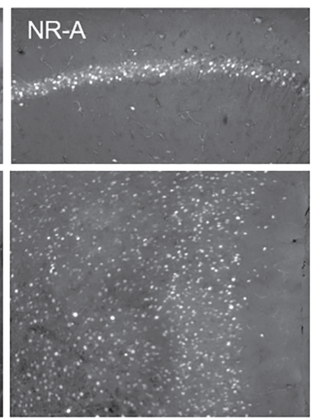
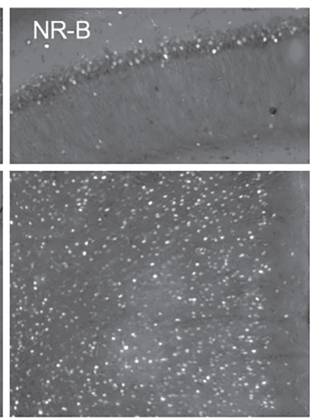

B
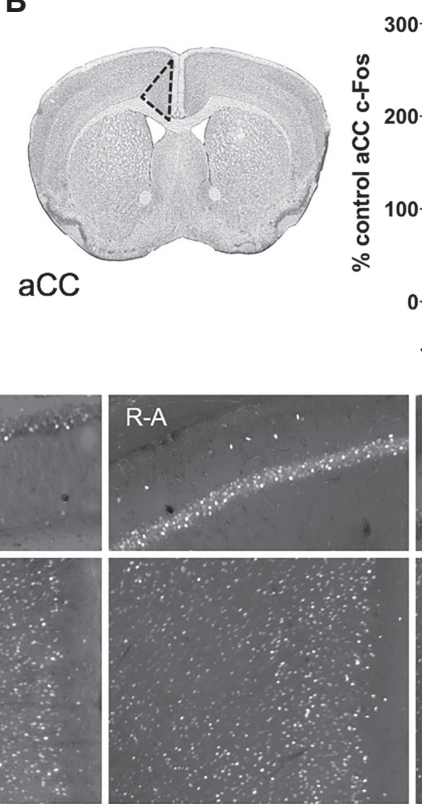
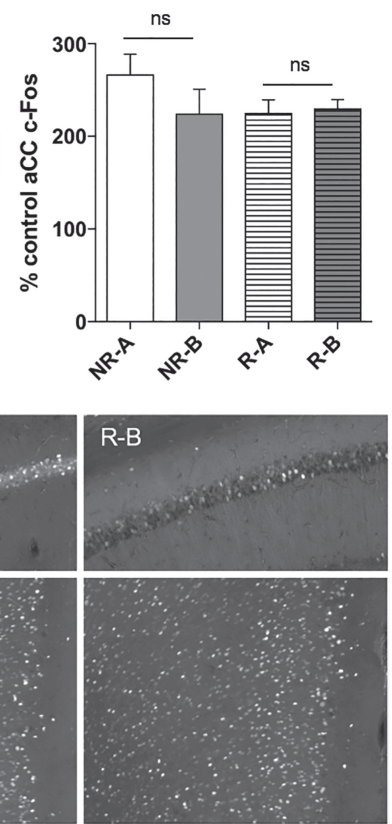

Figure 2. c-Fos expression is sensitive to retrieval context in dorsal hippocampus but insensitive to context and reminder in the aCC. ( $A$ ) (Left) Coronal section identifying the dCA1 of the hippocampus (outlined in black dashed box). (Right): Mean c-Fos expression levels in dCA1 were significantly higher when tested in Context-A than in Context-B. C-Fos expression levels following test in each context did not differ between the reminded (R) and nonreminded (NR) groups. Values are expressed as a percent change from the home cage control baseline c-Fos level. ( $B$ ) (Left) Coronal section identifying the aCC (outlined in black dashed box). (Right) Mean c-Fos expression levels in aCC did not differ between Context-A and Context-B test conditions, regardless of reminder condition. Values are expressed as a percent change from the home cage control baseline c-Fos level. Error bars represent the SEM. (*) $P<0.05$, ns $P>0.05$. (C) (Top) Representative c-Fos expression in the dCA1 for each experimental condition (NR-A, NR-B, R-A, R-B) and home cage control (HC) groups. Scale bar $=100 \mu \mathrm{m}$. Bottom: Representative c-Fos expression in the aCC for each experimental condition and home cage control groups. Scale bar $=100 \mu \mathrm{m}$. 
We replicated our group's previous findings in which a reminder in the conditioning context prior to remote memory testing reduced generalized freezing in a novel context. Rats subsequently tested in the conditioning context continued to display high levels of freezing, indicating that the brief reminder was an effective mnemonic cue that triggered retrieval of the context memory, while not inducing extinction learning in the conditioning context. In the absence of a reminder, high freezing levels were observed in both contexts. In line with our prediction, c-Fos was highly expressed in the dCA1 following Context-A testing, but not following Context-B testing, regardless of whether the context memory had been recently reactivated, indicating that the hippocampally mediated memory remains accessible at a remote time point, even in the absence of contextual cuing. The results of the reminder condition are consistent with the previously observed pattern of hippocampal activity observed when a context-specific memory is retrieved just $1 \mathrm{~d}$ following memory encoding (Sekeres et al. 2018a). It should be noted that the present study did not include a recent retrieval condition, and thus a comparison between these two timepoints should be interpreted cautiously.

Regarding activity in the aCC, two possible outcomes were proposed above. Consistent with the second outcome that predicted engagement of the reorganized remote memory trace during retrieval, the aCC was similarly activated following testing in both contexts, irrespective of reminder condition. Retrieval in either context may engage the aCC-mediated schematic type of the fear memory which represents the common elements of the event (Tse et al. 2011; Richards et al. 2014; Cullen et al. 2015; Kitamura et al. 2017; Sekeres et al. 2018b). While exposing the rat to the conditioning context prior to remote memory testing may engage the context-specific representation of the memory in hippocampus, it may also serve to activate the schematic representation of the event in the aCC. Even in the absence of pretest reactivation of this schema, testing in either the familiar or the novel context will similarly activate (or reactivate, when preceded by a reminder) this schematic representation. This may explain why the aCC continues to be highly activated in response to both contexts, while the hippocampus is only activated in response to the original conditioning context.

It should be noted that the high freezing observed in all groups during the Context-A reminder likely reflects retrieval of memory for the original conditioning experience, but it remains possible that a generalized representation activated by the reminder could also induce strong freezing behavior in Context-A. If the freezing during the reminder was driven by the generalized context memory representation, however, it is difficult to resolve why subsequent testing in novel Context-B would suddenly reengage context-specific memory retrieval during testing in these R-B animals (Fig. 1D).

These data support the position that a prefrontal-hippocampal network is engaged during remote context memory retrieval in the intact brain. It is likely that the context-specific memory persists, and a generalized schematic memory develops over time. Though both of these memory representations can coexist, the cues prior to retrieval determine which type dominates memory expression. Reminders were effective in allowing the contextspecific representation of the memory to become reinstated and expressed, with retrieval engaging both the hippocampus and aCC. These results are in line with previous findings that retrieval of a remote context fear memory, without reminders, does not require the hippocampus and generalizes to new contexts (Winocur et al. 2007; Goshen et al. 2011). Activity within the dCA1, however, continues to be required for retrieval of a precise, but not a generalized, remote context memory (Wiltgen et al. 2010), as occurs when a reminder is given. There is evidence suggesting that the ventral hippocampus and its projections to the medial prefrontal cortex, are required for the retrieval of the remote generalized context. Inactivation of the aCC and vHPC at this time prevents the expression of the generalized context memory, while leaving the context-specific memory intact (Cullen et al. 2015; Ortiz et al. 2019). While vHPC activity was not assessed in the present study, the results provide an interesting avenue for follow up studies investigating the role of the vHPC to determine how reactivating the remote memory via a Context-A reminder may alter the remote systems reconsolidation of generalized/schematic memories in the absence of a functional aCC and vHPC.

There is evidence that the same dCA1 neurons engaged during encoding continue to be recruited during remote memory retrieval (Tayler et al. 2013; Kitamura et al. 2017), supporting the idea that the hippocampus does not disengage during systems consolidation as a memory ages, while the aCC continues to develop a schematic representation of the memory that is insensitive to test context (Kitamura et al. 2017). Further, Einarsson et al. (2015) found that suppression of both the dorsal hippocampus and aCC was required to impair retrieval of a reactivated remote contextspecific memory, indicating that even for reactivated remote memory, either structure is sufficient to support retrieval.

These results also support recent evidence for chemogenetic activation of mPFC neurons tagged during encoding of a context fear memory being sufficient to induce freezing in a novel context at a remote time point (Matos et al. 2019). The originally tagged medial prefrontal cortical (mPFC, including the aCC) neurons are those recruited during remote memory retrieval in the novel context, and this systems-level transformation of the context memory is dependent upon the plasticity-related transcription factor c-AMP response-element binding (CREB) protein function in the mPFC. In line with the findings of Matos et al. (2019), Sekeres et al. (2012) previously showed that a remote generalized context memory can regain context-specificity following a reminder by enhancing CREB protein in the dorsal hippocampus during the remote memory reconsolidation period (Sekeres et al. 2012). The present data further support this idea, that in the intact brain, both structures come online during retrieval of the remote memory, and the recently reactivated context memory.

The results leave open the question of what neural mechanisms are involved in enabling the expression of context-specific memory with a reminder, but not without one. The data suggest that hippocampal cellular reconsolidation processes continue to be engaged following reactivation of a remote context fear memory. While context reactivation may cue retrieval of the hippocampally mediated context specific memory, the aCC which develops schematic memory representations over time, continues to be activated under normal physiological conditions during retrieval, but its ability to control behavior in the new context is suppressed. This suppression may result from the direct influence of the hippocampus on aCC output, or via a third mediating structure, such as the nucleus reunions (Xu and Südhof 2013; Dolleman-van der Weel et al. 2019), that coordinates activity between both of them. In their review, Tonegawa et al. (2018) also promote the coexistence of a remote episodic-like memory trace supporting the original conditioning context, and the development of a remote schematic memory trace in the $\mathrm{mPFC}$, although they concede that the precise network associated with the schematic memory trace has yet to be identified. Given that we did not tag cells active during encoding, our observational study cannot confirm whether the neurons engaged during encoding were the same neurons engaged during remote retrieval in the conditioning context. Based on the findings of Kitamura et al. (2017), it is possible that mPFC neurons originally involved in fear memory acquisition are the same cells that come online to support retrieval in the original context at a remote timepoint, while a different population of $\mathrm{mPFC}$ schema cells come to support retrieval of the generalized context 
memory at this remote timepoint, but this remains speculative. Thus, it still remains an open question as to the nature of the network supporting retrieval of the remote schematic memory, as well as the reactivated remote memory. Our findings provide a basis for further studies using more sophisticated labeling techniques to interrogate the neural underpinnings of remote and reconsolidated schematic context memories.

\section{Competing interest statement}

The authors declare no competing financial interests.

\section{Acknowledgments}

We gratefully acknowledge the technical assistance of Jeremy Audia, Christy Cole-Harding, Lana Madi, Josh Learned, and Yao-Fang Tan. This study was supported by an NSERC grant to G.W. (\#A8181) and CIHR grant to G.W. and M.M. (MOP125958). M.J.S. was supported by a CIHR post-doctoral fellowship.

Author contributions: Conceptualization: M.J.S., M.M., C.G., G.W.; Methodology: M.J.S., M.M., G.W.; Investigation: M.J.S., D.G.S.; Writing_Original Draft: M.J.S.; Writing-Review Editing: M.J.S., M.M., C.G., D.G.S., G.W.; Funding Acquisition: G.W., M.M.

\section{References}

Cole C, Josselyn SA. 2008. Transcription regulation of memory: CREB, CaMKIV, Fos/Jun, CBP, and SRF. In Learning and memory: a comprehensive reference (ed. Sweatt JD, Byrne JH), pp. 547-566. Elsevier, Oxford.

Cruz FC, Rubio FJ, Hope BT. 2015. Using c-fos to study neuronal ensembles in corticostriatal circuitry of addiction. Brain Res 1628: 157-173. doi:10 1016/j.brainres.2014.11.005

Cullen PK, Gilman TL, Winiecki P, Riccio DC, Jasnow AM. 2015. Activity of the anterior cingulate cortex and ventral hippocampus underlie increases in contextual fear generalization. Neurobiol Learn Mem 124: 19-27. doi:10.1016/j.nlm.2015.07.001

Debiec J, LeDoux JE, Nader K. 2002. Cellular and systems reconsolidation in the hippocampus. Neuron 36: 527-538. doi:10.1016/S0896-6273(02) 01001-2

DeNardo LA, Liu CD, Allen WE, Adams EL, Friedmann D, Fu L, Guenthner CJ, Tessier-Lavigne M, Luo L. 2019. Temporal evolution of cortical ensembles promoting remote memory retrieval. Nat Neurosci 22: 460. doi:10.1038/s41593-018-0318-7

Dolleman-van der Weel MJ, Griffin AL, Ito HT, Shapiro ML, Witter MP, Vertes RP, Allen TA. 2019. The nucleus reuniens of the thalamus sits at the nexus of a hippocampus and medial prefrontal cortex circuit enabling memory and behavior. Learn Mem 26: 191-205. doi:10.1101/ $\operatorname{lm} .048389 .118$

Einarsson EÖ, Nader K. 2012. Involvement of the anterior cingulate cortex in formation, consolidation, and reconsolidation of recent and remote contextual fear memory. Learn Mem 19: 449-452. doi:10.1101/lm 027227.112

Einarsson EÖ, Pors J, Nader K. 2015. Systems reconsolidation reveals a selective role for the anterior cingulate cortex in generalized contextual fear memory expression. Neuropsychopharmacology 40: 480-487. doi:10 $.1038 /$ npp.2014.197

Frankland PW, Bontempi B, Talton LE, Kaczmarek L, Silva AJ. 2004. The involvement of the anterior cingulate cortex in remote contextual fear memory. Science 304: 881-883. doi:10.1126/science.1094804

Goshen I, Brodsky M, Prakash R, Wallace J, Gradinaru V, Ramakrishnan C, Deisseroth K. 2011. Dynamics of retrieval strategies for remote memories. Cell 147: 678-689. doi:10.1016/j.cell.2011.09.033

Guzowski JF, Setlow B, Wagner EK, McGaugh JL. 2001. Experience-dependent gene expression in the rat hippocampus after spatial learning: a comparison of the immediate-early genes Arc, c-fos, and zif268. J Neurosci 21: 5089-5098. doi:10.1523/JNEUROSCI .21-14-05089.2001

Hardt O, Nadel L. 2018. Systems consolidation revisited, but not revised: the promise and limits of optogenetics in the study of memory. Neurosci Lett 680: 54-59. doi:10.1016/j.neulet.2017.11.062
Kitamura T, Ogawa SK, Roy DS, Okuyama T, Morrissey MD, Smith LM, Redondo RL, Tonegawa S. 2017. Engrams and circuits crucial for systems consolidation of a memory. Science 356: 73-78. doi:10.1126/science .aam6808

Matos MR, Visser E, Kramvis I, van der Loo RJ, Gebuis T, Zalm R, Rao-Ruiz P, Mansvelder HD, Smit AB, van den Oever MC. 2019. Memory strength gates the involvement of a CREB-dependent cortical fear engram in remote memory. Nat Commun 10: 2315. doi:10.1038/s41467019-10266-1

Morgan JI, Cohen DR, Hempstead JL, Curran T. 1987. Mapping patterns of c-fos expression in the central nervous system after seizure. Science 237: 192-197. doi:10.1126/science.3037702

Ortiz S, Latsko MS, Fouty JL, Dutta S, Adkins JM, Jasnow AM. 2019. Anterior cingulate cortex and ventral hippocampal inputs to the basolateral amygdala selectively control generalized fear. J Neurosci 39: 6526-6539. doi:10.1523/JNEUROSCI.0810-19.2019

Paxinos GW, Watson C. 1997. The rat brain in stereotaxic coordinates. Elsevier Academic Press, Amsterdam, Netherlands.

Richards BA, Xia F, Santoro A, Husse J, Woodin MA, Josselyn SA, Frankland PW. 2014. Patterns across multiple memories are identified over time. Nat Neurosci 17: 981. doi:10.1038/nn.3736

Sekeres MJ, Sargin D, Mercaldo V, Frankland PW, Josselyn SA. 2012. Increasing CRTC1 function in the dentate gyrus during memory formation or reactivation increases memory strength without compromising memory quality. J Neurosci 32: 17857-17868. doi:10 .1523/JNEUROSCI.1419-12.2012

Sekeres MJ, Moscovitch M, Winocur G, Anderson JAE, Pishdadian S, Wojtowicz JM, St-Laurent M, McAndrews MP, Grady C. 2018a. Changes in patterns of neural activity underlie a time-dependent transformation of memory across species. Hippocampus 20: 745-764. doi:10.1002/hipo .23009

Sekeres MJ, Winocur G, Moscovitch M. 2018b. The hippocampus and related neocortical structures in memory transformation. Neurosci Lett 680: 39-53. doi:10.1016/j.neulet.2018.05.006

Tayler KK, Tanaka KZ, Reijmers LG, Wiltgen BJ. 2013. Reactivation of neural ensembles during the retrieval of recent and remote memory. Curr Biol 23: 99-106. doi:10.1016/j.cub.2012.11.019

Tonegawa S, Morrissey MD, Kitamura T. 2018. The role of engram cells in the systems consolidation of memory. Nat Rev Neurosci 19: 485. doi:10 .1038/s41583-018-0031-2

Tse D, Takeuchi T, Kakeyama M, Kajii Y, Okuno H, Tohyama C, Bito H, Morris RG. 2011. Schema-dependent gene activation and memory encoding in neocortex. Science 333: 891-895. doi:10.1126/science .1205274

Vetere G, Kenney JW, Tran LM, Xia F, Steadman PE, Parkinson J, Josselyn SA, Frankland PW. 2017. Chemogenetic interrogation of a brain-wide fear memory network in mice. Neuron 94: 363-374.e364. doi:10.1016/j .neuron.2017.03.037

Wheeler AL, Teixeira CM, Wang AH, Xiong X, Kovacevic N, Lerch JP, McIntosh AR, Parkinson J, Frankland PW. 2013. Identification of a functional connectome for long-term fear memory in mice. PLoS Comput Biol 9: e1002853. doi:10.1371/journal.pcbi.1002853

Wiltgen BJ, Silva AJ. 2007. Memory for context becomes less specific with time. Learn Mem 14: 313-317. doi:10.1101/lm.430907

Wiltgen BJ, Zhou M, Cai Y, Balaji J, Karlsson MG, Parivash SN, Li W, Silva AJ. 2010. The hippocampus plays a selective role in the retrieval of detailed contextual memories. Curr Biol 20: 1336-1344. doi:10.1016/j.cub.2010 .06 .068

Winocur G, Moscovitch M, Sekeres M. 2007. Memory consolidation or transformation: context manipulation and hippocampal representations of memory. Nat Neurosci 10: 555-557. doi:10.1038/ nn1880

Winocur G, Frankland PW, Sekeres M, Fogel S, Moscovitch M. 2009. Changes in context-specificity during memory reconsolidation: selective effects of hippocampal lesions. Learn Mem 16: 722-729. doi:10 $.1101 / \mathrm{lm} .1447209$

Winocur G, Sekeres MJ, Binns MA, Moscovitch M. 2013. Hippocampal lesions produce both nongraded and temporally graded retrograde amnesia in the same rat. Hippocampus 23: 330-341. doi:10.1002/hipo .22093

Xu W, Südhof TC. 2013. A neural circuit for memory specificity and generalization. Science 339: 1290-1295. doi:10.1126/science.1229534

Received June 17, 2020; accepted in revised form October 15, 2019. 


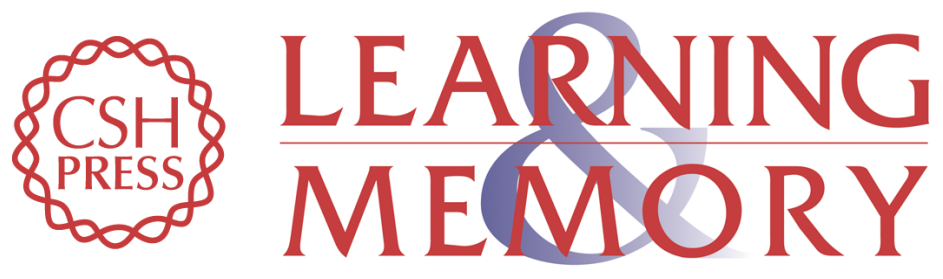

\section{Reminders reinstate context-specificity to generalized remote memories in rats: relation to activity in the hippocampus and aCC}

Melanie J. Sekeres, Morris Moscovitch, Cheryl L. Grady, et al.

Learn. Mem. 2020, 27:

Access the most recent version at doi:10.1101/lm.050161.119

Supplemental
Material http://learnmem.cshlp.org/content/suppl/2019/12/10/27.1.1.DC1

References This article cites 30 articles, 12 of which can be accessed free at: http://learnmem.cshlp.org/content/27/1/1.full.html\#ref-list-1

Creative This article, published in Learning \& Memory, is available under a Creative Commons Commons License (Attribution-NonCommercial 4.0 International), as described at License http://creativecommons.org/licenses/by-nc/4.0/.

Email Alerting Receive free email alerts when new articles cite this article - sign up in the box at the Service top right corner of the article or click here. 